\title{
Monotone comparative statics in games with non-monotonic best-replies: Contests and Cournot oligopoly*
}

\author{
Adriana Gama ${ }^{\dagger} \quad$ David Rietzke ${ }^{\ddagger}$
}

March 29, 2019

\begin{abstract}
In this paper, we employ lattice-theoretic techniques to derive a number of comparative statics in a logit contest - a class of games for which best-replies are generically non-monotonic. Using the same approach, we obtain several comparative statics in a Cournot oligopoly model without imposing the usual assumptions that ensure the game is one of strategic substitutes. Our analysis illustrates how latticetheoretic techniques can deliver powerful insights in games with nonmonotonic best-replies.
\end{abstract}

Keywords: Contests, Supermodularity, Cournot Oligopoly, Monotone Comparative Statics

JEL Classifications: C61, C72, D72

*A previous draft of this paper was circulated under the title, "Robust Comparative Statics in Contests".

${ }^{\dagger}$ Center of Economic Studies at El Colegio de México

${ }^{\ddagger}$ Lancaster University Management School. 


\section{Introduction}

Thanks to works such as Topkis (1978), Vives (1990, 1999), and Milgrom and Roberts (1990), lattice-theoretic techniques are used extensively in the economics literature. These tools allow researchers to obtain comparative statics results without the strong assumptions needed to apply the Implicit Function Theorem. Although these tools have proved extremely useful, their use in games has mainly been restricted to those with monotonic best-replies. Thus far, this has precluded their application in important classes of games - such as contests - which simply cannot be games of strategic complements/substitutes (Acemoglu and Jensen, 2013). Other games, including Cournot oligopoly, require assumptions on demand and/or costs that ensure globally monotonic best-replies (see Vives, 1999, for an overview). These assumptions rule out important classes of inverse demand curves frequently used in applications, such as hyperbolic demand.

In this paper, we show how lattice-theoretic tools (developed originally only for games of strategic complements/substitutes) are useful more generally. We first apply these tools in a (symmetric) contest under the logit contest success function (CSF). Contests are one important class of models that cannot yield monotone best-replies, and for which the existing literature (e.g. Dixit, 1987, Nti, 1997) has relied exclusively on the Implicit Function Theorem. We show that by focusing on subsets of the strategy space, the tools from supermodular games/games of strategic complementarities can be applied to obtain a strong regularity property on the best-response correspondence. This allows us to derive a number of comparative statics, which hold whenever a pure-strategy equilibrium exists. We then illustrate how our approach proves useful in other games that have non-monotonic best replies. To do so, we examine a Cournot oligopoly model. We obtain several comparative statics without relying on the Implicit Function Theorem or imposing the usual assumptions (e.g., logconcavity of inverse demand) that guarantee monotonic best-replies (see, e.g., Amir, 1996; Vives, 1999, 2005).

The logit CSF generalizes the model popularized by Tullock (1980), and is 
one of the most commonly used CSFs in the literature.1 The regularity property we establish on the best-response extends a finding from Dixit (1987), while our comparative statics extend/clarify results in Nti (1997), and complement Vives (2005), Acemoglu and Jensen (2013) and Jensen (2016). Dixit (1987) and Nti (1997) impose decreasing returns to scale on the contest technology, and invoke the Implicit Function Theorem. Contrasting Nti's (1997) results, when the contest technology exhibits increasing returns to scale, we show that equilibrium payoffs may be strictly decreasing in the value of the prize, and that equilibrium aggregate activity may be decreasing in the number of players. Vives (2005) studies a patent race model, which relates closely to the logit-contest. He shows that under very mild conditions, the game is strictly log-supermodular, thus yielding upward sloping best replies. The logit-contest, in contrast, generally has non-monotonic best-replies, and requires a different approach. Acemoglu and Jensen (2013) and Jensen (2016) allow asymmetries between players and exploit tools from aggregative games.

We also provide conditions that ensure existence of a pure-strategy equilibrium in the logit contest. For these results, we exploit an equivalence with the Cournot oligopoly model, and apply findings from Amir (1996) and Amir and Lambson (2000). These results complement the analyses of Szidarovszky and Okuguchi (1997), Cornes and Hartley (2005), Yamakazi (2008), Acemoglu and Jensen (2013) and Jensen (2016). These studies consider asymmetric contests, but impose stronger assumptions on the returns-to-scale of the contest technology.2 ${ }^{2}$

\section{The logit contest}

We consider a contest in which $n$ symmetric players compete for a single prize of common value, $V$. Each player, $i \in\{1, \ldots, n\}$, chooses an effort, $e_{i} \in \mathbb{R}_{+}$, according to the cost function, $C: \mathbb{R}_{+} \rightarrow \mathbb{R}_{+}$. If player $i$ chooses effort $e_{i}$, and

\footnotetext{
${ }^{1}$ See Corchón (2007) and Konrad (2009) for overviews of the contest literature. See Skaperdas (1996) for an overview of the logit CSF.

2 Cornes and Hartley (2005) allow for increasing returns to scale in the special case of the Tullock CSF.
} 
the other $n-1$ players choose efforts according to $\mathbf{e}_{-i} \in \mathbb{R}_{+}^{n-1}$, the probability that $i$ wins is given by the logit CSF:

$$
P\left(e_{i}, \mathbf{e}_{-i}\right)=\frac{\phi\left(e_{i}\right)}{R+\sum_{j=1}^{n} \phi\left(e_{j}\right)} .
$$

We refer to $\phi: \mathbb{R}_{+} \rightarrow \mathbb{R}_{+}$as the production function, and we call $\phi\left(e_{i}\right)$ player $i$ 's "force". The parameter, $R \geq 0$, is referred to as the "discount rate". Following Tullock (1980), much of the contest literature sets $R=0$; however, in settings such as patent races or inducement contests, it may happen that no player wins. $R>0$ captures this possibility (see, e.g. Loury, 1979). In the context of a patent race, an increase in $R$ can then be interpreted as a decrease in the likelihood that one player obtains the patent. In order to avoid discontinuities in the payoff function and simplify our proofs, we will rule out the case $R=0$ and assume $R>0$, but we discuss the case where $R=0$ in Section 2.3. When $\phi(x)=x^{r}$, and $R=0$, the logit CSF corresponds to the popular Tullock CSF. The expected payoff to player $i$ is,

$$
\hat{\pi}_{i}\left(e_{i}, \mathbf{e}_{-i}\right)=\frac{\phi\left(e_{i}\right)}{R+\sum_{j=1}^{n} \phi\left(e_{j}\right)} V-C\left(e_{i}\right) .
$$

Player $i$ chooses $e_{i}$ to maximize $\pi_{i}$, taking $\mathbf{e}_{-i}$ as given. We make the following assumptions:

\section{Assumption 1.}

(i) $\phi$ is continuous and strictly increasing.

(ii) $C$ is lower semi continuous and increasing.

(iii) For all $\mathbf{e}_{-i} \in \mathbb{R}_{+}^{n-1}, \lim _{e \rightarrow \infty} \hat{\pi}\left(e, \mathbf{e}_{-i}\right)<0$

Assumptions 1(1)-(ii) imply that greater effort strictly increases a player's likelihood of winning, but at a greater cost to the player. We do not require continuity of $C$, which has relevant economic content, as lower semi continuity allows for (avoidable) fixed costs. We also do not require $\phi(0)=0$ or $C(0)=0$, 
which allows for the possibility of past sunk investments in effort. Assumption 1)(iii) implies that we may, without further loss of generality, restrict attention to effort choices $e \in[0, \bar{e}]$ for some arbitrarily large $\bar{e}>0$.

\section{A Recasting}

Let $x \equiv \phi(e), y \equiv \sum_{j \neq i} \phi\left(e_{j}\right)$, and $z \equiv x+y$ denote, respectively, the force allocated to the contest by some player $i$, the total force of all players other than $i$, and the total force. Let $\underline{x}=\phi(0), \bar{x}=\phi(\bar{e}), \underline{y}=(n-1) \underline{x}$, and $\bar{y}=(n-1) \bar{x}$. We can then think of the players as choosing forces directly. Since $\phi$ is strictly increasing and continuous, the inverse function, $\phi^{-1}:[\underline{x}, \bar{x}] \rightarrow[\underline{e}, \bar{e}]$, is welldefined, strictly increasing, and continuous. We let $\kappa=C \circ \phi^{-1}$.

To facilitate some comparative statics, we let $\kappa$ be parameterized by some $\theta \in \mathbb{R}$, such that if player $i$ 's force is $x$, her total cost is $\kappa(x, \theta)$. Note that this leaves open the possibility that $\theta$ enters the original model through $\phi$ and/or $C$. Assume that $\kappa$ is strictly submodular in $(x, \theta)$ : for $x^{\prime \prime}>x^{\prime}$ and $\theta^{\prime \prime}>\theta^{\prime}$,

$$
\kappa\left(x^{\prime \prime}, \theta^{\prime \prime}\right)-\kappa\left(x^{\prime}, \theta^{\prime \prime}\right)<\kappa\left(x^{\prime \prime}, \theta^{\prime}\right)-\kappa\left(x^{\prime}, \theta^{\prime}\right)
$$

If $\kappa$ is twice differentiable in $e$ and $\theta$, then the condition above is implied by $\kappa_{x \theta}<0$. That is, an increase in $\theta$ strictly decreases the marginal cost of effort. We can then write player $i$ 's payoff as,

$$
\pi(x, y)=\frac{x}{R+x+y} V-\kappa(x, \theta) .
$$

Since the game is symmetric, we avoid the use of subscripts for ease of notation. For any $y \geq 0$ we let

$$
X^{*}(y)=\arg \max \{\pi(x, y) \mid x \in[\underline{x}, \bar{x}]\},
$$

and let $r: \mathbb{R}_{+} \rightarrow[\underline{x}, \bar{x}]$ denote an arbitrary single-valued selection from $X^{*}$. In our definition of $X^{*}$ we allow $y$ to take on any positive value, including those outside the feasible range of $[\underline{y}, \bar{y}]$. We can thus think of $X^{*}$ as an extension of the best-response correspondence; but in a slight abuse of terminology, we 
refer to it simply as the best-response. Our assumptions on $\phi$ and $C$ ensure that, for any fixed $y \geq 0, \pi(\cdot, y)$ is upper semi continuous; since the choice set is compact, $X^{*}(y)$ is non empty (and possibly set-valued).

We divide our results into two sets. For our first set of results - in Section 2.1 - we impose no additional structure on the model. Although the existence of a pure-strategy equilibrium cannot be guaranteed, our results apply immediately in any setting where a symmetric pure-strategy equilibrium exists. For our second set of results - in Section 2.2 - we provide conditions that ensure existence of a pure-strategy equilibrium, and study how aggregate behavior depends on the number of players. We discuss the case where $R=0$ in Section 2.3. All proofs are contained in the Appendix.

We focus on pure-strategy Nash equilibria; for brevity we simply write "equilibrium". We refer to $\phi$ and $C$ jointly as the contest technology. We say that the technology exhibits decreasing [increasing] returns to scale if $\kappa(\cdot, \theta)$ is convex [concave]. Throughout this paper, when we write "increasing" or "decreasing" we mean in the weak sense; otherwise we shall write "strictly increasing" or "strictly decreasing".

Finally, borrowing terminology from Jensen (2016), we say $i$ is an "absolute favorite to lose [win]" if $\frac{x}{R+x+y}<[>] \frac{1}{2}$; equivalently, $x<[>] y+R$. We let $\Phi_{L}=\left\{(x, y) \in[\underline{x}, \bar{x}] \times \mathbb{R}_{+} \mid x<y+R\right\}$ denote the subset of the strategy space in which $i$ is an absolute favorite to lose, and let $\Phi_{W}=\left\{(x, y) \in[\underline{x}, \bar{x}] \times \mathbb{R}_{+} \mid x>\right.$ $y+R\}$ denote the subset in which $i$ is an absolute favorite to win.

\subsection{Comparative statics}

In this section we explore how symmetric equilibrium behavior varies with the parameters of the model; namely, $n, V$, and $R$. A symmetric equilibrium satisfies $x^{*} \in X^{*}\left(y^{*}\right)$ and $x^{*}=\frac{y^{*}}{n-1}$. Note that since $X^{*}(y)$ is defined for all $y \in \mathbb{R}_{+}$, we need to ensure that the candidate equilibrium value of $y$ corresponds to a feasible strategy profile for the other players. But since $x^{*} \in X^{*}\left(y^{*}\right)$ implies $x^{*} \in[\underline{x}, \bar{x}]$, clearly, $y^{*}=(n-1) x^{*}$ is feasible.

We begin by establishing a regularity property of players' best-response 
correspondences. The following lemma is useful in doing so.

Lemma 1. Let $x^{\prime \prime}>x^{\prime} \geq \underline{x}$ and $y^{\prime \prime}>y^{\prime} \geq 0$. If $x^{\prime \prime} x^{\prime}<[>]\left(y^{\prime \prime}+R\right)\left(y^{\prime}+R\right)$. Then,

$$
\pi\left(x^{\prime \prime}, y^{\prime \prime}\right)-\pi\left(x^{\prime \prime}, y^{\prime}\right)<[>] \pi\left(x^{\prime}, y^{\prime \prime}\right)-\pi\left(x^{\prime}, y^{\prime}\right)
$$

It is well-known that no CSF can be supermodular or submodular in own and rivals' efforts over its entire domain. To illustrate, suppose $n=2$ and let $P$ denote the CSF. If the contest is decisive (i.e., one of the two players will win the contest). $]^{3}$ then for all $e_{1}$ and $e_{2}$ it holds: $P\left(e_{1}, e_{2}\right)+P\left(e_{2}, e_{1}\right) \equiv 1$. Suppose $P$ is twice differentiable, then, $P_{12}\left(e_{1}, e_{2}\right)=-P_{12}\left(e_{2}, e_{1}\right)$. It is easy to see that this implies whenever player 1's objective function is supermodular in $\left(e_{1}, e_{2}\right)$, then player 2's objective function is submodular, and vice versa. Yet, Lemma 1 implies that, under the logit CSF, $\pi$ is strictly submodular/supermodular over a subset of the strategy space. Specifically, $\pi$ is strictly submodular on $\Phi_{L}$ and strictly supermodular on $\Phi_{W}$.

Now, using the Implicit Function Theorem, Dixit (1987) (also see Jensen, 2016) shows that when $i$ 's rivals increase their aggregate action, $i$ responds aggressively to defend her position (by increasing her action) when she is a favorite to win, and responds timidly (by reducing her action) when she is a favorite to lose. Using Lemma 1, our next result follows almost immediately by Topkis' Theorem 4 and it extends this regularity property found by Dixit (1987).

Lemma 2. Suppose Assumption 1 is satisfied. Let $y^{\prime \prime}>y^{\prime} \geq 0$, and let $x^{\prime \prime} \in X^{*}\left(y^{\prime \prime}\right)$ and $x^{\prime} \in X^{*}\left(y^{\prime}\right)$. If $x^{\prime \prime} x^{\prime}<\left(y^{\prime \prime}+R\right)\left(y^{\prime}+R\right)$ then $x^{\prime \prime} \leq x^{\prime}$. If $x^{\prime \prime} x^{\prime}>\left(y^{\prime \prime}+R\right)\left(y^{\prime}+R\right)$ then $x^{\prime \prime} \geq x^{\prime}$.

Lemma 2 It implies that every selection from $X^{*}$ is increasing [decreasing] when it is fully contained in $\Phi_{W}\left[\Phi_{L}\right]$. Figure 1 illustrates a typical bestresponse. $\Phi_{W}$ is the region above the dashed line, $x=y+R$, and $\Phi_{L}$ is the region below this line. Note that in a symmetric equilibrium, $x=\frac{y}{n-1}<y+R$,

\footnotetext{
${ }^{3}$ Under the logit CSF, when $R>0$, the contest is not decisive. Nevertheless, a similar logic applies.

${ }^{4}$ See, e.g., Theorem 3.1 in Vives (1990) or Theorem A.1 in Amir and Lambson (2000).
} 
which means any symmetric equilibrium occurs in $\Phi_{L} .5^{5}$ Since each selection from the best-response is decreasing in this region, and the "equilibrium line", $x=\frac{y}{n-1}$, is strictly increasing, there can be, at most, one point of intersection between the two. Thus any symmetric equilibrium must be unique. Our next result states this observation formally.

Proposition 1. Suppose Assumption 1 is satisfied. For a fixed $n \geq 2$, if a symmetric equilibrium exists, it must be unique.

Also observe in Figure 1 that an increase in $n$ rotates the equilibrium line downwards. It is clear from the figure that equilibrium individual force must decrease, while the total force of $i$ 's rivals must increase. Our next result formalizes these observations, and also shows how payoffs vary with $n$. In what follows, we let $x_{t}$ denote the symmetric equilibrium force when the parameter of interest is equal to $t$, and analogously define $y_{t}, z_{t}$, and $\pi_{t}$.

Proposition 2. Suppose Assumption 1 is satisfied. For a fixed $n \geq 2$, in a symmetric equilibrium, with $n^{\prime \prime}>n^{\prime}$,

(i) The individual force (and effort) is decreasing in the number of players: $x_{n^{\prime \prime}} \leq x_{n^{\prime}}$.

(ii) The other players' joint force is increasing in the number of players: $y_{n^{\prime \prime}} \geq y_{n^{\prime}}$.

(iii) The expected per-player payoff is decreasing in the number of players: $\pi_{n^{\prime \prime}} \leq \pi_{n^{\prime}}$.

Consistent with other results that invoke lattice-theoretic techniques, our findings only ensure weak monotonicity of individual forces/efforts and payoffs in $n$. The following example shows that strict monotonicity cannot be guaranteed in general.

\footnotetext{
${ }^{5}$ Note that if $R=0$ and $n=2$, this is no longer true, as the lines $x=y+R$ and $x=\frac{y}{n-1}$, coincide. We discuss this in Section 2.3 .
} 


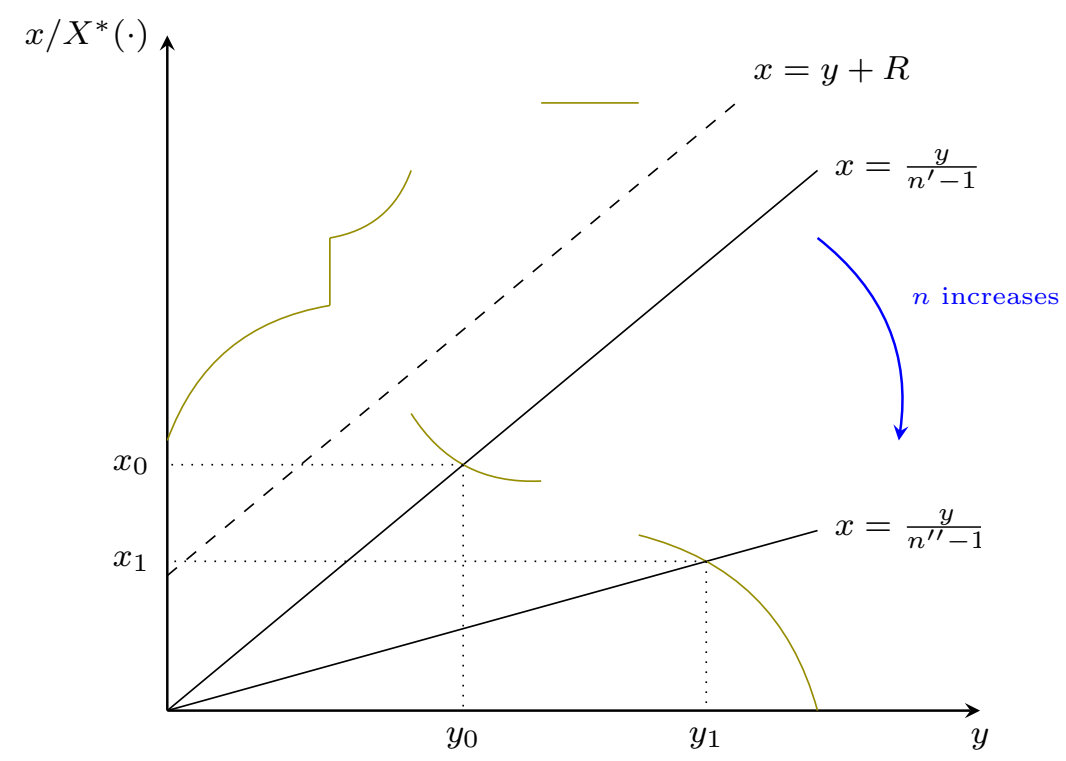

Figure 1: An example of a typical best-response correspondence (in green). Each selection from $X^{*}$ must be increasing in the region above the line $x=y+R$, and decreasing in the region below this line.

Example 1. Let $V=10, R=0]^{6} \underline{x}=0$, and $\kappa(x)=\left\{\begin{array}{ll}\sqrt{x} & x \leq 1 \\ (x+1)^{2}-3 & x \geq 1\end{array}\right.$. For each $n=2, \ldots, 10$ there is a unique symmetric equilibrium in which all players choose $x^{*}=1$.

We next study how symmetric equilibrium behavior depends on the other parameters of the model, namely, the value of the prize, $V$, the discount rate, $R$, and the cost parameter, $\theta$.

Proposition 3. Fix $n \geq 2$ and suppose Assumption 1 is satisfied. Then in a symmetric equilibrium,

(i) Individual and total forces/efforts are increasing in the value of the prize:

For $V^{\prime \prime}>V^{\prime}, x_{V^{\prime \prime}} \geq x_{V^{\prime}}$ and $z_{V^{\prime \prime}} \geq z_{V^{\prime}}$.

\footnotetext{
${ }^{6}$ The main idea in this example would also hold for $R$ strictly positive but sufficiently small.
} 
(ii) Individual and total forces/efforts are increasing in the cost parameter: For $\theta^{\prime \prime}>\theta^{\prime}, x_{\theta^{\prime \prime}} \geq x_{\theta^{\prime}}$ and $z_{\theta^{\prime \prime}} \geq z_{\theta^{\prime}}$.

(iii) Individual and total forces/efforts are decreasing in the discount rate: For $R^{\prime \prime}>R^{\prime}, x_{R^{\prime \prime}} \leq x_{R^{\prime}}$ and $z_{R^{\prime \prime}} \leq z_{R^{\prime}}$

Parts (i) and (ii) of Proposition 3 follow from the fact that $\pi$ is strictly supermodular in $(x, V)$ and $(x, \theta)$. By Topkis' Theorem, an increase in either of these parameters shifts a player's best response upward, and leads to an increase in individual force/effort in any symmetric equilibrium. Figure 2 illustrates: Following an increase in $V$ or $\theta$, the player's best response shifts up from the green to the blue curve, and the symmetric equilibrium increases from the point $E_{0}$ to the point $E_{1}$.

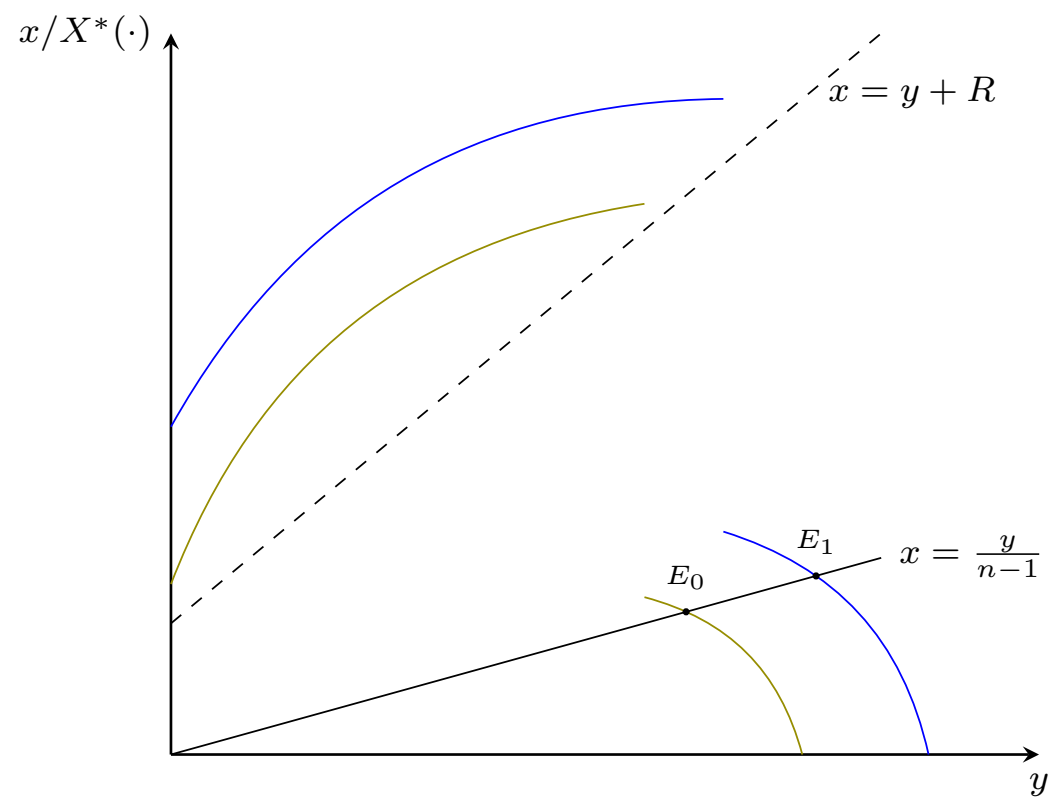

Figure 2: An illustration of the impact of an increase in $V$ or $\theta$ on the best response correspondence. The green curve represents the best response before the parameter increase; the blue curve represents the best response after the parameter increase. Following the parameter change, the symmetric equilibrium increases from $E_{0}$ to $E_{1}$.

To understand part (iii) of Proposition 3 , first note that $\pi$ depends on $R$ and $y$ insofar as it depends on the sum, $y+R$. Adapting Lemmas 1 and 2 , it 
is straightforward to show that, for any fixed $y \geq 0, \pi$ is strictly submodular in $(x, R)$ on $\Phi_{L}$, and that any selection from $X^{*}$ is decreasing in $R$ whenever $X^{*}$ is contained in $\Phi_{L}$. So, suppose $R$ increases from $R^{\prime}$ to $R^{\prime \prime}$. When a player's best response is contained in $\Phi_{L}$ (for both parameter values), this portion of the best response must shift down, following the increase in the parameter. Figure 3 illustrates; the green curve is the best response when $R=R^{\prime}$, and the blue curve is the best response when $R=R^{\prime \prime}>R^{\prime}$. In the region between the lines, $x=y+R^{\prime}$ and $x=y+R^{\prime \prime}$, the relationship between the two best-response functions is difficult to ascertain in general. In the region below the line $x=y+R^{\prime}$, there is a clear ordering between the two, with the best-response shifting down following the increase in the parameter. As any symmetric equilibrium occurs below this line, we can conclude that the symmetric equilibrium force decreases.

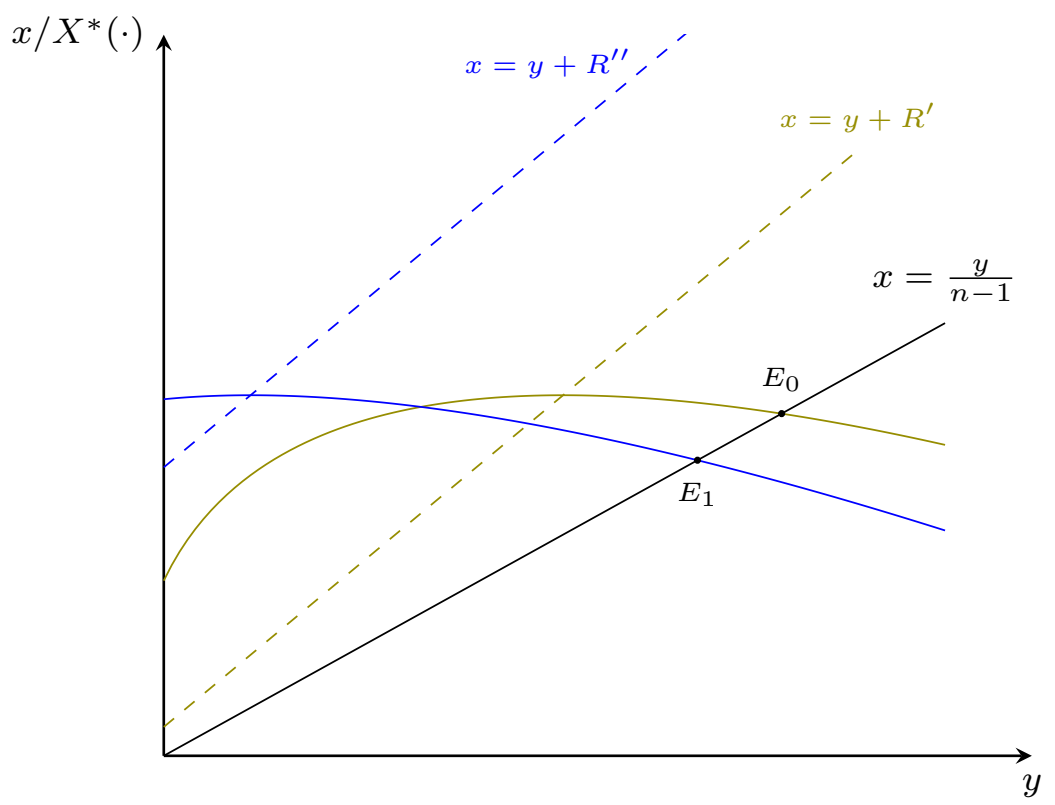

Figure 3: An illustration of the impact of a change in $R$ on the best response. The green curve is the best response when $R=R^{\prime}$; the blue curve is the best response when $R=R^{\prime \prime}>R^{\prime}$. Following an increase in $R$, the equilibrium decreases from $E_{0}$ to $E_{1}$.

We now explore the relationship between the parameters of the model, and equilibrium payoffs. When the contest technology exhibits decreasing returns 
to scale, Nti (1997) shows that equilibrium payoffs increase in $V$. The next example shows that this result does not hold in general. Indeed, equilibrium per-player payoffs may be strictly decreasing in the prize or the cost parameter, $\theta$.

Example 2. Let $n=2, R=0,7 \underline{x}=0$, and

$$
\kappa(x, \theta)= \begin{cases}\frac{x}{\theta} & x \leq 1 \\ \frac{x^{\alpha}}{\alpha \theta}+\frac{\alpha-1}{\alpha \theta} & x \geq 1\end{cases}
$$

If $1 \leq \frac{V \theta}{4} \leq \frac{1-\alpha}{1-2 \alpha}$, the symmetric equilibrium per-player force is $x^{*}=\left(\frac{V \theta}{4}\right)^{\frac{1}{\alpha}}$. The equilibrium per-player payoff is

$$
\pi^{*}=\frac{1-\alpha}{\alpha \theta}-V\left(\frac{1-2 \alpha}{4 \alpha}\right) .
$$

For $0<\alpha<\frac{1}{2}, \pi^{*}$ is is strictly decreasing in $V$ for fixed $\theta$, and strictly decreasing in $\theta$ for fixed $V$. For instance, if $\alpha=\frac{1}{3}$ and $\theta=1$ then for $4 \leq V \leq 8, x^{*}=\left(\frac{V}{4}\right)^{3}$, and $\pi^{*}=2-\frac{V}{4}$. If $\alpha=\frac{1}{3}$ and $V=1$ then for $4 \leq \theta \leq 8, x^{*}=\left(\frac{\theta}{4}\right)^{3}$ and $\pi^{*}=\frac{2}{\theta}-\frac{1}{4}$.

There are two competing forces acting on a player $i$ 's payoff following an increase in the prize. There is a direct positive effect, since the value of winning increases. But there is also an indirect negative effect, since $i$ 's rivals increase their forces. Nti (1997) shows that the direct positive effect outweighs the indirect negative effect when the contest technology exhibits decreasing returns to scale. With decreasing returns, higher levels of force are produced at greater marginal cost, which dampens the indirect effect. For technologies with increasing returns, a contrasting logic applies: Players' optimal efforts are more sensitive to changes in the prize, and the indirect effect is exacerbated.8

\footnotetext{
${ }^{7}$ The main idea in this example would also hold for $R$ strictly positive but sufficiently small.

${ }^{8}$ This effect is evident in Example 2, as $\frac{\partial^{2} x^{*}}{\partial \alpha \partial V}<0$. That is, a decrease in $\alpha$, which leads to more pronounced increasing returns to scale, implies that $x^{*}$ is more sensitive to changes in the prize.
} 
As Example 2 shows, with increasing returns it may well be that the indirect effect dominates the direct effect. A similar intuition holds for changes in $\theta$.

While the relationship between equilibrium per-player payoffs and $V / \theta$ is ambiguous, our next result shows that there is a clear relationship between payoffs and $R$.

Proposition 4. Suppose Assumption 1 is satisfied. In a symmetric equilibrium, the per-player expected payoff is decreasing in the discount rate: For $R^{\prime \prime}>R^{\prime}, \pi_{R^{\prime}} \geq \pi_{R^{\prime \prime}}$.

As with an increase in $V$ or $\theta$, there are two competing forces acting on equilibrium payoffs following a decrease in $R$. There is a direct positive effect, since a decrease in $R$ increases the likelihood of a player winning the prize. But there is an indirect negative effect caused by the increase in the efforts of one's rivals (see Proposition 3(iii)). As it happens, the positive effect always outweighs the negative effect.

Although $y$ is decreasing in $R$, the proof of Proposition 4 shows that the sum, $y+R$, is increasing in $R$. To see why, consider a decrease in $R$ from $R^{\prime \prime}$ to $R^{\prime}$. Figure 4 illustrates the impact of the parameter change: The green curve is (a portion of) the best response when $R=R^{\prime \prime}$, and the blue curve is the best response when $R=R^{\prime}<R^{\prime \prime}$. When the parameter is $R^{\prime \prime}$, the total equilibrium force of $i$ 's rivals is $y^{\prime \prime}$. Now since $X^{*}$ depends only on the sum, $y+R$, an increase in $R$ shifts the best response outwards by a horizontal distance equal to $R^{\prime \prime}-R^{\prime}$. As $x^{\prime \prime}$ is a best response to $y^{\prime \prime}$ when $R=R^{\prime \prime}$, then $x^{\prime \prime}$ must also be a best response to $\hat{y}=y^{\prime \prime}+R^{\prime \prime}-R^{\prime}$ when $R=R^{\prime}$. Using the monotonicity of the best response in the region relevant for equilibrium, it is clear from the figure that any point of intersection between the blue best response and the line $x=\frac{y}{n-1}$ must occur at a value $y^{\prime} \in\left[y^{\prime \prime}, \hat{y}\right]$. This means $y^{\prime}+R^{\prime} \leq y^{\prime \prime}+R^{\prime \prime}$. Using this fact, it is straightforward to show that a player's equilibrium payoff must increase following a decrease in $R .9$

\footnotetext{
${ }^{9}$ Figure 4 illustrates a scenario in which $y+R$ strictly decreases in equilibrium following the decrease in $R$. But this need not be the case: Lemma 2 does not preclude the possibility that $\hat{x}=\frac{\hat{y}}{n-1}$ is also a best response to $\hat{y}$ when $R=R^{\prime}$ (although this would not be possible in the way we've drawn the figure).
} 


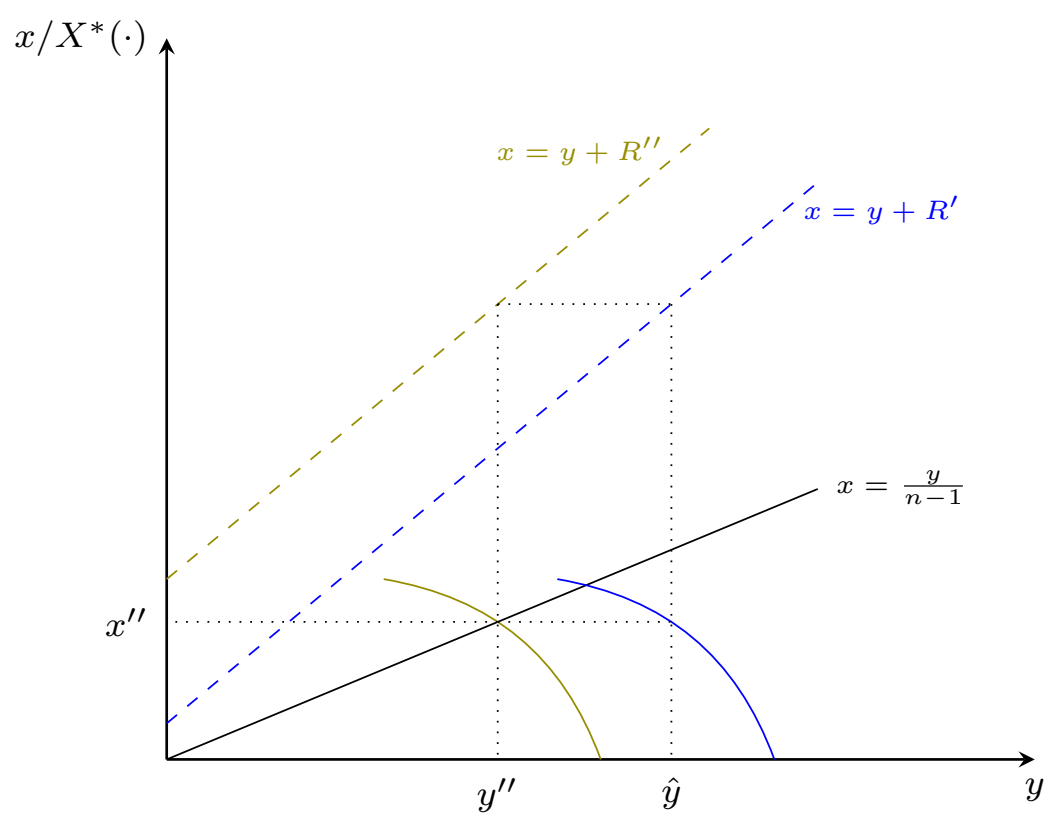

Figure 4: An illustration of the impact of a change in $R$ on (a portion of) the best response correspondence. The green curve is the best-response when $R=R^{\prime \prime}$; the blue curve is the best response when $R=R^{\prime}<R^{\prime \prime}$.

\section{Summarizing}

The comparative statics results provided in Propositions 2 - 4 extend results in Nti (1997). We have shown that neither decreasing returns to scale nor differentiability are essential drivers of these results. Rather, the structure imposed by the logit CSF yields payoff functions that exhibit strict increasing [decreasing] differences over $\Phi_{W}\left[\Phi_{L}\right]$. This feature drives the regularity property of the best response found by Dixit (1987), which we extend in Lemma 2. From this property, and the fact that any symmetric equilibrium occurs in $\Phi_{L}$, the comparative statics follow. But Example 2 shows that the assumption of decreasing returns to scale is an important driver of the typical finding that equilibrium payoffs increase in the value of the prize. 


\subsection{Equilibrium existence and effects of entry}

We next turn to the question of existence, and study how aggregate behavior varies with the number of players. These results rely on the equivalence between our contest and a symmetric Cournot oligopoly with inverse demand, $\frac{V}{R+Q}$, and cost function $\kappa$. The results in this section are consequences of our Proposition 1, and results in Amir and Lambson (2000). We strengthen Assumption 1 as follows:

\section{Assumption 2.}

(i) For all $e>0, \phi(e)$ is twice continuously differentiable with $\phi^{\prime}(e)>0$. Moreover, $\phi(0)=0$.

(ii) For all $e \geq 0, C(e)$ is twice continuously differentiable with $C^{\prime}(e) \geq 0$.

We do not wish to rule out the Tullock CSF, which is not differentiable at zero when $r<1$. For this reason, we do not require $\phi(0)$ to be differentiable; Assumption 2 is otherwise consistent with assumptions in Amir and Lambson (2000).

Noting that $z=x+y$, a player's payoff can be expressed, $\tilde{\pi}(z, y)=$ $\frac{z-y}{R+z} V-\kappa(z-y)$. We can then think of some player $i$ as choosing the total force, taking the total force of the other players as given. That is, player $i$ solves, $\max \{\tilde{\pi}(z, y) \mid \bar{x}+y \geq z \geq y\}$. Assumption 2 ensures that $\kappa(x)$ is twice continuously differentiable for all $x>0$. For all $z>y$, let $\Delta(z, y)$ denote the cross partial derivative of $\tilde{\pi}$ with respect to $z$ and $y: \Delta(z, y)=\frac{V}{(R+z)^{2}}+\kappa^{\prime \prime}(z-y)$. Note that $\Delta$ is defined on the lattice, $\Phi=\left\{(z, y) \in \mathbb{R}_{+} \mid(n-1) \bar{x} \geq y \geq\right.$ $0, y+\bar{x} \geq z>y\}$. As in Amir and Lambson (2000), the sign of $\Delta$ on $\Phi$ plays a critical role in our analysis. When $\Delta>[<] 0$ on $\Phi, \tilde{\pi}$ is strictly supermodular [submodular] on its domain. First we consider the case where $\Delta>0$ on $\Phi$.

Proposition 5. In addition to Assumptions 1](iii) and 2, suppose $\Delta>0$ on $\Phi$. Then,

(i) For each $n \in \mathbb{N}$, there exists a unique symmetric equilibrium, and no asymmetric equilibria. 
(ii) The equilibrium total force is increasing in $n$ : For $n^{\prime \prime}>n^{\prime}, z_{n^{\prime \prime}} \geq z_{n^{\prime}}$.

Proposition 5 provides conditions under which the existence of a unique symmetric equilibrium can be guaranteed, and also shows that equilibrium total force is increasing in the number of players. The existence result and the comparative static with respect to $n$ follows from Amir and Lambson (2000), ${ }^{10}$ while uniqueness follows by Proposition 1. The condition, $\Delta>0$, limits the returns to scale of the contest technology, but also depends on the magnitudes of the discount rate and the prize. Jensen (2016) provides a result similar to Proposition 5 in an asymmetric contest. In the special case where all players are symmetric, Jensen's (2016) Assumption 3 amounts to, $\frac{C^{\prime \prime}}{C^{\prime}} \geq \frac{\phi^{\prime \prime}}{\phi^{\prime}}$, which is equivalent to $\kappa^{\prime \prime} \geq 0 .{ }^{11}$ Note that $\Delta>0$ if $\kappa^{\prime \prime} \geq 0$, but $\Delta$ may be strictly positive even if $\kappa$ is strictly concave everywhere (see Example 3 in Gama and Rietzke, 2017).

We now consider the case where $\Delta<0$. Our next two results are consequences of Amir and Lambsons (2000) Theorems 2.5 and 2.6, respectively.

Proposition 6. In addition to Assumptions 1 and 2, suppose $\Delta<0$ on $\Phi$. Then, for any $n \in \mathbb{N}$,

(i) For any $m<n$, if a symmetric equilibrium exists in the m-player contest (with individual force $x_{m}$, say) then the following configuration constitutes an equilibrium for the $n$ player contest: Each of any $m$ players chooses force $x_{m}$ while the remaining $n-m$ players exert zero effort. In particular, an n-player equilibrium always exists in which one player chooses the optimal single-player effort and the other $n-1$ players choose zero effort.

(ii) A unique symmetric equilibrium exists if, for each $y \in[0, \bar{y}], \pi(\cdot, y)$ is strictly quasiconcave.

\footnotetext{
${ }^{10}$ Note, moreover, that $\Delta>0$ is a special case of Acemoglu and Jensen's (2013) "uniform local solvability" condition, which could also be applied to show the comparative static with respect to $n$.

${ }_{11}^{11}$ Jensen $\mathrm{s}(2016 \mathrm{~s})$ Assumption 3 is a sufficient condition for the uniform solvability condition to be satisfied. Relaxing Assumption 3 and instead imposing uniform local solvability, would be equivalent to imposing $\Delta>0$.
} 
(iii) No other equilibrium other than those described in parts (i) and (ii) can exist.

When $\Delta<0$ over its domain, several asymmetric equilibria may exist: ${ }^{12}$ moreover, there always exists an equilibrium in which $n-1$ players are inactive. An equilibrium with a single active player is not standard in contests. Note that the condition, $\Delta<0$ requires $\kappa^{\prime \prime}<0$ and $R>0$. It should be clear that an equilibrium with only one active player could never exist if $R=0$.

Our next result shows how total equilibrium forces vary with the number of players when $\Delta<0$.

Proposition 7. In addition to Assumptions 1 and 2, suppose $\Delta(z, y)<0$ on $\Phi$. Then,

(i) Under the hypothesis of Proposition 6(i), all the asymmetric equilibria for all $m<n$ are invariant in the number of players $n$.

(ii) Under the hypothesis of Proposition 6(ii), the total equilibrium force, $z_{n}$, is decreasing in $n$.

Proposition 7(i) clearly holds since, in the asymmetric equilibria with $m<$ $n$, an additional player exerts no effort. Proposition 7 (ii) contrasts much of the work in contests under the logit CSF ${ }^{13}$ The intuition is akin to that of a natural monopoly. The condition $\Delta<0$ implies strong increasing returns to scale. Limiting entry allows a player to take full advantage of her increasing returns. Example 4 in Gama and Rietzke (2017) illustrates our finding.

Related to Proposition 7, Baye et al. (1993) show that total effort increases if the player with the highest value is excluded in an asymmetric all-pay auction. Intuitively, the high-value player discourages effort from the other players; removing this player "levels the playing field", and encourages greater

\footnotetext{
12 Perez-Castrillo and Verdier (1992) and Cornes and Hartley (2005) find a similar equilibrium structure under the Tullock CSF with $r>1$. Chowdhury and Sheremeta (2011) also show the potential for multiple asymmetric equilibria under the Tullock CSF when the size of the prize varies with efforts.

${ }^{13}$ One exception is Amegashie (1999). Under the Tullock CSF, Amegashie (1999) shows that total effort may be decreasing in $n$, when a player's prize includes a fixed component, and a variable component, which increases linearly in a player's effort.
} 
effort among the remaining players. In contrast to the Exclusion Principal, our result applies in a symmetric contest, so there is no scope for leveling the playing field. However, with increasing returns to scale, the addition of another player can have a strong discouragement effect.14

\subsection{The case, $\mathrm{R}=0$}

Since much of the work in the contest literature utilizing the logit CSF assumes $R=0$, in this section we briefly discuss this case. The formal results discussed herein can be found in Gama and Rietzke (2017). Our results in Section 2.1 rely heavily on the fact that any symmetric equilibrium occurs in $\Phi_{L}$. But when $R=0$ and $n=2$, this is no longer true. In this case, multiple symmetric equilibria may exist (at most 2); but uniqueness is restored if $n>2$ or $\kappa$ is differentiable. Regardless, our comparative statics in Section 2.1 continue to hold if one replaces "in a symmetric equilibrium" with "in the smallest and largest symmetric equilibria". Proposition 5 also holds when $R=0$. However, the condition $\Delta<0$, which is relevant for Propositions 6 and 7 , requires $R>0$.

\section{Cournot oligopoly}

Beyond our contribution to the contest literature, this paper adds to the literature on monotone comparative statics more broadly. Our approach in the contest demonstrates that lattice-theoretic techniques - originally developed for games of strategic complements/substitutes - can deliver powerful insights in games with non-monotonic best-replies. In this section we apply our approach to a symmetric Cournot oligopoly model. We will not impose the usual assumptions that ensure globally monotonic best-replies, nor will we rely on the Implicit Function Theorem.

Let $P: \mathbb{R}_{+} \rightarrow \mathbb{R}$ denote inverse demand and let $C: \mathbb{R}_{+} \times \mathbb{R} \rightarrow \mathbb{R}_{+}$denote each firm's cost function. To facilitate some comparative statics, we suppose

\footnotetext{
${ }^{14}$ The choice of CSF plays a critical role in driving the Exclusion Principle. For instance, when marginal cost is constant, it is well-known that the result does not hold under the Tullock CSF (See, e.g., Fang, 2002, Matros, 2006; Menicucci, 2006).
} 
that $P$ is parameterized by some $R \geq 0$ such that, if total output is $z$, the market price is $p=P(z+R)$, where $P$ is strictly decreasing. $R$ is a demandshift parameter; an increase in this parameter decreases the market price for a given market output, $z$. Further, we assume $C$ is parameterized by $\theta \in \mathbb{R}$ such that if a firm produces $x$, total cost is given by $C(x, \theta)$. We assume $P$ and $C$ are twice continuously differentiable with $P^{\prime}<0, C_{x} \geq 0$, and $C_{x \theta}<0$. Thus, an increase in $\theta$ decreases marginal cost. We impose differentiability for ease of exposition, but is not needed for our results.

If firm $i$ chooses output, $x$, and the total output of the other $n-1$ firms is $y$, the payoff to firm $i$ is $\pi(x, y)=x P(x+y+R)-C(x, \theta)$. We assume for all $y, \lim _{x \rightarrow \infty} \pi(x, y)<0$, which implies that we may restrict attention to $x \in[0, \bar{x}]$ for some arbitrarily large $\bar{x} \in \mathbb{R}_{++}$. Note that our assumptions on $\pi$ ensure that a firm's best response correspondence is non-empty for each $y$.

Following the structure of our previous results, we first provide several comparative statics assuming existence of a symmetric equilibrium; we then discuss conditions from the literature under which such an equilibrium is guaranteed to exist. In what follows, we let $x_{t}$ denote a per-firm symmetric equilibrium output level when the parameter of interest is equal to $t$ and define $y_{t}$, and $\pi_{t}$ analogously. We also let $z_{t}=x_{t}+y_{t}$ denote total equilibrium output.

Proposition 8. Consider a symmetric Cournot oligopoly. Suppose there exists $n_{0} \geq 2$ such that,

$$
\text { for all } z \geq 0, \quad P^{\prime}(z)+\frac{z}{n_{0}} P^{\prime \prime}(z)<0 .
$$

Then, if for $n \geq n_{0}$ a symmetric equilibrium exists, it must be unique. Moreover, in any symmetric equilibrium with $n \geq n_{0}$ firms,

(i) For $n^{\prime \prime}>n^{\prime} \geq n_{0}, x_{n^{\prime \prime}} \leq x_{n^{\prime}}, y_{n^{\prime \prime}} \geq y_{n^{\prime}}$, and $\pi_{n^{\prime \prime}} \leq \pi_{n^{\prime}}$.

(ii) For $R^{\prime \prime}>R^{\prime}, x_{R^{\prime \prime}} \leq x_{R^{\prime}}, z_{R^{\prime \prime}} \leq z_{R^{\prime}}$, and $\pi_{R^{\prime \prime}} \leq \pi_{R^{\prime}}$.

(iii) For $\theta^{\prime \prime}>\theta^{\prime}, x_{\theta^{\prime \prime}} \geq x_{\theta^{\prime}}$ and $z_{\theta^{\prime \prime}} \geq z_{\theta^{\prime}}$.

The main implication of condition (3) is that $\pi$ is strictly submodular in $(x, y)$ on $\Phi_{0}=\left\{(x, y) \in \mathbb{R}_{+}^{2} \mid x \leq \frac{y+R}{n_{0}-1}\right\}$. It follows that every selection of a 
firm's best response is decreasing when contained in $\Phi_{0}$. For $n \geq n_{0}$ a symmetric equilibrium satisfies $x=\frac{y}{n-1} \leq \frac{y+R}{n_{0}-1}$, and therefore must be contained in $\Phi_{0}$. Precisely the same arguments used in the proofs of Propositions 1 4 can then be applied. Note that, as previously mentioned, differentiability is imposed only for ease of exposition. One could alternatively replace condition (3) with the assumption that $\pi$ is strictly submodular on $\Phi_{0}$, and the conclusion of Proposition 8 would hold.

Our next result in this section shows that the hypothesis of Proposition 8 is always satisfied if $P^{\prime}$ is bounded away from 0 and $P^{\prime \prime}$ is bounded away from $+\infty$.

Proposition 9. If there exists $\epsilon>0$ and $B<\infty$ such that, $\left|P^{\prime}\right|>\epsilon$ and $P^{\prime \prime}<B$, then the hypothesis of Proposition 8 is satisfied.

Turning to existence of equilibrium, Theorem 2.7 in Amir and Lambson (2000) shows that an equilibrium exists so long as $\tilde{\Delta}(x, z)=P^{\prime}(z)-C^{\prime \prime}(x) \neq 0$ for all $z \geq x \geq 0$. Although asymmetric equilibria may exist, in any of these equilibria all active firms (those producing non-zero output) must produce the same level of output. So, under the condition $\tilde{\Delta} \neq 0$, our Proposition 8 applies in any equilibrium of the Cournot game with $m \geq n_{0}$ active firms. Alternatively, Theorem 2.1 in Amir and Lambson (2000) shows that a symmetric equilibrium exists and no asymmetric equilibria exist if $\tilde{\Delta}>0$. Under this condition, our Proposition 8 implies that for $n \geq n_{0}$, this equilibrium is unique.

\section{Discussion}

The standard approach to comparative statics relies on first-order conditions together with assumptions sufficient to ensure the validity of the Implicit Function Theorem (see, e.g., Seade, 1980; Novshek, 1980). Following this approach and temporarily ignoring the integer constraint on $n$, one could show that if $\tilde{\Delta}>0$ then, a condition similar to condition (4) would imply $\frac{d x_{n}}{d n}<0$ (see, e.g. Vives, 2008, Proposition 4). However, as noted by Vives (2005), this approach is fraught with shortcomings. In particular, it leads to ambiguous conclusions 
if $\tilde{\Delta}<0$; moreover, it delivers no meaningful results in the presence of fixed costs (implying non-differentiable cost functions).

The alternative approach, using lattice-theoretic techniques, relies on conditions sufficient to ensure globally monotone best responses (see Vives, 1999. 2005, for overviews). Consider the following condition:

$$
\text { For all } z \geq 0, P^{\prime}(z)+z P^{\prime \prime}(z)<0 \text {. }
$$

Under Condition (4) the Cournot game is a strictly submodular game, and therefore every selection from the best response is globally decreasing. Novshek (1985) used condition (4) to prove existence of an equilibrium (without imposing symmetry). Vives (1990) later established the link between Novshek s (1985) result and the theory of supermodular games. Acemoglu and Jensen (2013) extend Novshek's (1985) finding to a general class of submodular aggregative games and derive a number of comparative statics (see also, Jensen, 2010). Amir (1996) provides an alternative condition - namely, log-concavity of inverse demand - which implies that the Cournot game is ordinally submodular and therefore is one of strategic substitutes.

Hyperbolic inverse demand is one example of a commonly used functional form, which is log-convex and also does not satisfy (4). If $P$ is log-convex and $C \equiv 0$, then the game is log-supermodular and best replies are globally increasing (Amir, 1996). Amir and Lambson (2000) uses this property to show that extremal equilibrium per-firm outputs increase with entry. Otherwise, with log-convex inverse demand and arbitrary costs, it might appear as though one is relegated to rely on the Implicit Function Theorem for comparative statics. Indeed, prior analyses of the logit contest (which is a special case of the Cournot model with hyperbolic demand) have relied on this approach. Our approach, on the other hand, offers useful and powerful insights for this type of application. Example 3 illustrates.

Example 3. Let $P(z)=(z+R)^{-\gamma}$, where $\gamma>0$ and $R>0$. Let $C$ be any (non-trivial) cost function. For any $n>\gamma+1$, and $z \geq 0$, we have $P^{\prime}(z)+\frac{z}{n} P^{\prime \prime}(z)=\frac{\gamma[z(1+\gamma-n)-n R]}{n(z+R)^{2+\gamma}}<0$. Proposition 8 applies and therefore, if $a$ 
symmetric equilibrium exists in the game with $n^{\prime \prime}>n^{\prime} \geq \gamma+1$ firms, it must be unique; moreover, $x_{n^{\prime \prime}} \leq x_{n^{\prime}}$.

We close this section by noting the relationship between our Proposition 8 and results on large markets in the Cournot oligopoly (Novshek, 1980; Bamón and Frayssé, 1985; Vives, 1999, Sec. 4.4). In a market with $r$ consumers, each with demand curve $D(p)$, the market inverse demand will be $P_{r}(z)=P\left(\frac{z}{r}\right)$. Under relatively mild conditions on inverse demand, when $r$ is sufficiently large, the analogue of condition (4) will hold. In consequence, best-replies will be monotonically decreasing (globally); existence and a number of comparative statics can be then be obtained using known results. Our condition (3) is in the same spirit, but is conceptually quite distinct from these replication

results. For one, under condition (3) $n$ sufficiently large is not enough to ensure existence of a pure strategy equilibrium. Second, our condition does not imply globally monotone best-replies even when $n$ is sufficiently large. Our condition, instead, ensures monotonicity of best-replies only on a restricted subset of the strategy space. Assuming a symmetric equilibrium exists, this is enough to generate meaningful comparative statics.

\section{Conclusion}

In this paper, we used lattice-theoretic techniques to derive a number of comparative statics results in a logit contest. To the best of our knowledge, this is the first paper to apply these tools in contests. We then obtained a number of comparative statics in a Cournot oligopoly model without relying on the Implicit Function Theorem, or imposing the usual assumptions that ensure global monotonicity of the best replies. Our approach illustrates how latticetheoretic techniques can be applied to obtain meaningful comparative statics results in games with non-monotonic best-replies. 


\section{Appendix}

\section{Proof of Lemma 1}

Let $x^{\prime \prime}>x^{\prime} \geq \underline{x}$ and $y^{\prime \prime}>y^{\prime} \geq 0$. Let $\tilde{y}^{\prime \prime}=y^{\prime \prime}+R$ and $\tilde{y}^{\prime}=y^{\prime}+R$. The reader can easily verify that expression $(2)$ is equivalent to

$$
\frac{x^{\prime \prime}}{x^{\prime \prime}+\tilde{y}^{\prime \prime}}-\frac{x^{\prime \prime}}{x^{\prime \prime}+\tilde{y}^{\prime}}<[>] \frac{x^{\prime}}{x^{\prime}+\tilde{y}^{\prime \prime}}-\frac{x^{\prime}}{x^{\prime}+\tilde{y}^{\prime}},
$$

which holds if and only if $x^{\prime \prime} x^{\prime}<[>] \tilde{y}^{\prime \prime} \tilde{y}^{\prime}$.

\section{Proof of Lemma 2}

We will show the first statement in the lemma; the proof of the second statement is analogous. Let $y^{\prime \prime}>y^{\prime} \geq 0, x^{\prime \prime} \in X^{*}\left(y^{\prime \prime}\right)$ and $x^{\prime} \in X^{*}\left(y^{\prime}\right)$. Suppose $x^{\prime \prime} x^{\prime}<\left(y^{\prime \prime}+R\right)\left(y^{\prime}+R\right)$. Proceed by contradiction: Suppose, contrary to the lemma, $x^{\prime \prime}>x^{\prime}$. Then by Lemma 1 .

$$
0 \leq \pi\left(x^{\prime \prime}, y^{\prime \prime}\right)-\pi\left(x^{\prime}, y^{\prime \prime}\right)<\pi\left(x^{\prime \prime}, y^{\prime}\right)-\pi\left(x^{\prime}, y^{\prime}\right) \leq 0
$$

where the l.h.s. inequality follows since $x^{\prime \prime} \in X^{*}\left(y^{\prime \prime}\right)$, and the r.h.s. inequality follows since $x^{\prime} \in X^{*}\left(y^{\prime}\right) .15$ We have a contradiction; hence it must be that $x^{\prime \prime} \leq x^{\prime}$

\section{Proof of Proposition 1}

Fix $n \geq 2$. We show that if a symmetric equilibrium exists, it must be unique. Let $x_{1}$ and $x_{2}$ be two symmetric equilibrium levels of force, and let let $y_{i}=(n-$ 1) $x_{i}, i=1,2$. Proceed by contradiction, and suppose that these two equilibria are distinct; in particular, suppose $x_{1}>x_{2}$; equivalently, $y_{1}>y_{2}$. Note that for $i=1,2, x_{i}$ and $y_{i}$ satisfy: $x_{i} \in X^{*}\left(y_{i}\right)$ and $x_{i}=\frac{y_{i}}{n-1}$. But since $\frac{y_{i}}{n-1} \leq y_{i}$, it must hold that $x_{i}<y_{i}+R$ for $i=1,2$; hence, $x_{1} x_{2}<\left(y_{1}+R\right)\left(y_{2}+R\right)$.

\footnotetext{
${ }^{15}$ The choice set, $[\underline{x}, \bar{x}]$, is independent of $y$, so $x^{\prime}\left(x^{\prime \prime}\right)$ is certainly feasible when others' joint force is $y^{\prime \prime}\left(y^{\prime}\right)$.
} 
Since $y_{1}>y_{2}$ (by assumption), Lemma 2 implies $x_{1} \leq x_{2}$, which yields a contradiction. Therefore, the symmetric equilibrium must be unique.

\section{Proof of Proposition 2}

\section{Parts (i)-(ii)}

Fix $n^{\prime \prime}>n^{\prime}$, and suppose that a symmetric pure-strategy equilibrium exists in the $n^{\prime \prime}$ and $n^{\prime}$ player contests. From the arguments in the first part of this proof, we know that the symmetric equilibrium must be unique. Let $x^{\prime \prime}\left(x^{\prime}\right)$ denote the individual equilibrium individual force when the contest has $n^{\prime \prime}$ (respectively, $n^{\prime}$ ) players; let $y^{\prime \prime}=\left(n^{\prime \prime}-1\right) x^{\prime \prime}$ and $y^{\prime}=\left(n^{\prime}-1\right) x^{\prime}$.

We first show part (ii). First note that if $y^{\prime} \leq\left(n^{\prime \prime}-1\right) \underline{x}$, then as feasibility requires $y^{\prime \prime} \geq\left(n^{\prime \prime}-1\right) \underline{x}$, it follows that $y^{\prime \prime} \geq y^{\prime}$. Then suppose $y^{\prime}>\left(n^{\prime \prime}-1\right) \underline{x}$. We will show that there cannot be a symmetric equilibrium with $y<y^{\prime}$ when $n=n^{\prime \prime}$. Fix $y_{0} \in\left[\left(n^{\prime \prime}-1\right) \underline{x}, y^{\prime}\right)$, and let $x_{0} \in X^{*}\left(y_{0}\right)$. Clearly if $x_{0} \geq y_{0}+R$ then $x_{0}>\frac{y_{0}}{n^{\prime \prime}-1}$. If $x_{0}<y_{0}+R$, then Lemma 2 implies $x_{0} \geq x^{\prime}$. But since $n^{\prime \prime}>n^{\prime}$ and $y^{\prime}>y_{0}$ it holds, $x^{\prime}=\frac{y^{\prime}}{n^{\prime}-1}>\frac{y^{\prime}}{n^{\prime \prime}-1}>\frac{y_{0}}{n^{\prime \prime}-1}$; thus, $x_{0}>\frac{y_{0}}{n^{\prime \prime}-1}$. We have now established that for all $y \in\left[\left(n^{\prime \prime}-1\right) \underline{x}, y^{\prime}\right), x \in X^{*}(y)$ implies $x>\frac{y}{n^{\prime \prime}-1}$; thus, there cannot be a symmetric equilibrium with $y<y^{\prime}$ when $n=n^{\prime \prime}$. It follows that $y^{\prime \prime} \geq y^{\prime}$. This establishes part (ii).

Next, we show $x^{\prime \prime} \leq x^{\prime}$. We have already shown that $y^{\prime \prime} \geq y^{\prime}$. If $y^{\prime \prime}=y^{\prime}$ then $n^{\prime \prime}>n^{\prime}$, implies $x^{\prime \prime}=\frac{y^{\prime \prime}}{n^{\prime \prime}-1}<\frac{y^{\prime}}{n^{\prime}-1}=x^{\prime}$. If $y^{\prime \prime}>y^{\prime}$ then since $x^{\prime \prime}<y^{\prime \prime}+R$ and $x^{\prime}<y^{\prime}+R$, Lemma 2 implies $x^{\prime \prime} \leq x^{\prime}$. Since individual force decreases following the increase in $n$, clearly individual effort decreases as well. This establishes part (i).

\section{Part (iii)}

Let $\pi^{\prime \prime}\left(\pi^{\prime}\right)$ denote the equilibrium individual expected payoff in the contest with $n^{\prime \prime}$ (respectively, $n^{\prime}$ ) players where $n^{\prime \prime}>n^{\prime}$. We have the following string of inequalities: 


$$
\begin{aligned}
\pi^{\prime} & =\frac{x^{\prime}}{R+x^{\prime}+y^{\prime}} V-\kappa\left(x^{\prime}\right) \\
& \geq \frac{x^{\prime \prime}}{R+x^{\prime}+y^{\prime}} V-\kappa\left(x^{\prime \prime}\right) \\
& \geq \frac{x^{\prime \prime}}{R+x^{\prime \prime}+y^{\prime \prime}} V-\kappa\left(x^{\prime \prime}\right) \\
& =\pi^{\prime \prime}
\end{aligned}
$$

The first inequality holds by definition of $x^{\prime}$. The second inequality follows since, as we showed in part (ii), $y^{\prime \prime} \geq y^{\prime}$. This establishes part (iii) and the proposition.

\section{Proof of Proposition 3}

We prove parts (i) and (ii) jointly. Fix $n$, and let $t$ denote either the parameter $V$ or $\theta$. It is easily verified that $\pi$ is strictly supermodular in $t$ and $x$. By Topkis' Theorem (see, e.g. Topkis, 1978, or Theorem A.1 in Amir and Lambson $(2000)$ ), it follows that any selection, $r(\cdot)$, from $X^{*}$ is increasing in $t$ for each $y$. It is clear that this implies that any intersection between a player's reaction curve and the line $x=\frac{y}{n-1}$ must lie further from the origin following an increase in $t$. Since individual force increases, then individual effort and total force/effort must increase, as $n$ is fixed. This proves parts (i) and (iii).

Next, we show part (iii). Let $R^{\prime \prime}>R^{\prime}$, and suppose a symmetric equilibrium exists for both parameter values. Let $x^{\prime \prime}$ and $x^{\prime}$ denote the symmetric equilibrium individual force when the parameter is $R^{\prime \prime}$, respectively $R^{\prime}$. Let $y^{\prime \prime}=(n-1) x^{\prime \prime}$ and $y^{\prime}=(n-1) x^{\prime}$. Since $\pi$ depends on $y$ and $R$ insofar as it depends on the sum, $y+R, X^{*}$ depends only on this sum. We write $X^{*}(y+R)$ to denote the set of best-replies to $y$ when the parameter is $R$. Note that our result follows immediately if $x^{\prime \prime}=\underline{x}$; so, assume $x^{\prime \prime}>\underline{x}$; equivalently, $y^{\prime \prime}>\underline{y}$.

Let $\hat{y}=R^{\prime \prime}-R^{\prime}+y^{\prime \prime}>y^{\prime \prime}$. By construction, $\hat{y}+R^{\prime}=y^{\prime \prime}+R^{\prime \prime}$; therefore, $X^{*}\left(\hat{y}+R^{\prime}\right)=X^{*}\left(y^{\prime \prime}+R^{\prime \prime}\right)$. By definition of $x^{\prime \prime}$, this means $x^{\prime \prime} \in X^{*}\left(\hat{y}+R^{\prime}\right)$. We now show that there cannot be a symmetric equilibrium in which $y<y^{\prime \prime}$ when 
$R=R^{\prime}$. Fix $y_{0} \in\left[\underline{y}, y^{\prime \prime}\right)$, and let $x_{0} \in X^{*}\left(y_{0}+R^{\prime}\right)$. Clearly, if $x_{0}>y_{0}+R^{\prime}$ then $x_{0}>\frac{y_{0}}{n-1}$. If $x_{0} \leq y_{0}+R^{\prime}$, then since $x^{\prime \prime} \leq y^{\prime \prime}<\hat{y}+R^{\prime}$, it holds $x_{0} x^{\prime \prime}<\left(y_{0}+R^{\prime}\right)\left(\hat{y}+R^{\prime}\right)$. As, $x_{0} \in X^{*}\left(y_{0}+R^{\prime}\right), x^{\prime \prime} \in X^{*}\left(\hat{y}+R^{\prime}\right)$, and $\hat{y}>y^{\prime \prime}>y_{0}$, Lemma 2 implies $x_{0} \geq x^{\prime \prime}=\frac{y^{\prime \prime}}{n-1}>\frac{y_{0}}{n-1}$.

We have now shown that for all $y \in\left[\underline{y}, y^{\prime \prime}\right), x \in X^{*}\left(y+R^{\prime}\right)$ implies $x>\frac{y}{n-1}$; thus, there cannot exist a symmetric equilibrium in which $y<y^{\prime \prime}$ when $R=R^{\prime}$. It therefore must be that $y^{\prime} \geq y^{\prime \prime}$; equivalently, $x^{\prime} \geq x^{\prime \prime}$. Since individual force increases following a decrease in $R$, then individual effort and total force/effort must increase, as $n$ is fixed. This establishes part (iii) and the proposition.

\section{Proof of Proposition 4}

Let $R^{\prime \prime}>R^{\prime}$ and suppose that a symmetric equilibrium exists for both parameter values. Let $x^{\prime \prime}\left(x^{\prime}\right)$ denote the equilibrium per-player force when the parameter is $R^{\prime \prime}\left(R^{\prime}\right)$. Let $y^{\prime \prime}=(n-1) x^{\prime \prime}$ and $y^{\prime}=(n-1) x^{\prime}$. Finally, let $X^{*}(y+R)$ denote the best response to $y$ when the parameter is $R \cdot{ }^{16}$

Let $\hat{y}=y^{\prime \prime}+R^{\prime \prime}-R^{\prime}>y^{\prime \prime}$. As we showed in the proof of Proposition 3(ii), it must be that $x^{\prime \prime} \in X^{*}\left(\hat{y}+R^{\prime}\right)$. Moreover, since $x^{\prime \prime}=\frac{y^{\prime \prime}}{n-1}<\frac{\hat{y}}{n-1}<\hat{y}+R^{\prime}$, Lemma 2 implies that for all $y>\hat{y}$, if $x \in X^{*}\left(y+R^{\prime}\right)$ and $x<y+R^{\prime}$, then $x \leq x^{\prime \prime}<\frac{y}{n-1}$. Thus, there cannot exist a symmetric equilibrium with $y>\hat{y}$ when $R=R^{\prime}$. Therefore, $y^{\prime} \leq \hat{y}$, which implies $y^{\prime}+R^{\prime} \leq \hat{y}+R^{\prime}=y^{\prime \prime}+R^{\prime \prime}$. Next, let $\pi^{\prime \prime}\left(\pi^{\prime}\right)$ denote the equilibrium payoff to a player when the parameter is $R^{\prime \prime}$ (respectively, $R^{\prime}$ ). It holds,

$$
\begin{aligned}
\pi^{\prime} & =\frac{x^{\prime}}{x^{\prime}+y^{\prime}+R^{\prime}} V-\kappa\left(x^{\prime}\right) \\
& \geq \frac{x^{\prime \prime}}{x^{\prime \prime}+y^{\prime}+R^{\prime}} V-\kappa\left(x^{\prime \prime}\right) \\
& \geq \frac{x^{\prime \prime}}{x^{\prime \prime}+y^{\prime \prime}+R^{\prime \prime}} V-\kappa\left(x^{\prime \prime}\right) \\
& =\pi^{\prime \prime}
\end{aligned}
$$

\footnotetext{
${ }^{16}$ Recall from the proof of Proposition 3(ii) that a player's best response depends on $y$ and $R$ insofar as it depends on the sum, $y+R$.
} 
The first inequality follows by definition of $x^{\prime}$; the second follows since $y^{\prime \prime}+R^{\prime \prime} \geq y^{\prime}+R^{\prime}$. This establishes the proposition.

\section{Proof of Propositions $5-7$}

The contest is equivalent to a symmetric Cournot oligopoly with inverse demand function $\tilde{P}(z)=\frac{V}{R+z}$, and cost function, $\tilde{C}(x)=\kappa(x)$. The existence of a symmetric equilibrium, and non-existence of any asymmetric equilibria established in Proposition 5 follows from Theorem 2.1 in Amir and Lambson (2000). The uniqueness of this equilibrium follows from Proposition 1. Part (ii) of Proposition 5 follows immediately by Theorem 2.2(b) in Amir and Lambson (2000). Propositions 6 and 7 follow by Amir and Lambson's (2000)

Theorems 2.5 and 2.6, respectively.

\section{Proof of Proposition 8}

We first establish the following lemma.

Lemma 3. Fix $n_{0} \in \mathbb{N}$. If $P^{\prime}(z)+\frac{z}{n_{0}} P^{\prime \prime}(z)<0$ for all $z \geq 0$ then $\frac{\partial^{2}}{\partial x \partial y} \pi(x, y, R, \theta)<$ 0 for all $(x, y) \in \Phi_{0} \equiv\left\{\left(x^{\prime}, y^{\prime}\right) \in \mathbb{R}_{+}^{2} \mid 0 \leq x^{\prime} \leq \frac{y^{\prime}+R}{n_{0}-1}\right\}$.

Proof. Let $n_{0} \in \mathbb{N}$ be given, and suppose for all $z \geq 0, P^{\prime}(z)+\frac{z}{n_{0}} P^{\prime \prime}(z)<0$. Let $(x, y) \in \Phi_{0}$ be given, where $\Phi_{0}$ is as defined in the lemma. We want to show $\frac{\partial^{2}}{\partial x \partial y} \pi(x, y, R, \theta)=P^{\prime}(x+y+R)+x P^{\prime \prime}(x+y+R)<0$. If $P^{\prime \prime}(x+y+R) \leq 0$ then we are done; so, suppose $P^{\prime \prime}(x+y+R)>0$. Let $z=x+y+R$. We have the following string of inequalities:

$$
\begin{aligned}
0 & >P^{\prime}(z)+\frac{z}{n_{0}} P^{\prime \prime}(z) \\
& =P^{\prime}(x+y+R)+\frac{x+y+R}{n_{0}} P^{\prime \prime}(x+y+R) \\
& \geq P^{\prime}(x+y+R)+\frac{x+\left(n_{0}-1\right) x}{n_{0}} P^{\prime \prime}(x+y+R) \\
& =P^{\prime}(x+y+R)+x P^{\prime \prime}(x+y+R)
\end{aligned}
$$


The strict inequality holds by the supposition of the lemma. The first equality holds by our choice of $z$. The first weak inequality holds since $P^{\prime \prime}(x+$ $y)>0$ (by assumption) and since $(x, y) \in \Phi_{0}$ implies $x\left(n_{0}-1\right) \leq y+R$. The lemma follows.

Lemma 3 implies that, under the hypothesis of the proposition, $\pi$ is strictly submodular in $(x, y)$ on $\Phi_{0}=\left\{(x, y) \in \mathbb{R}_{+}^{2} \mid x \leq \frac{y+R}{n_{0}-1}\right\}$. By Topkis' Theorem, whenever the best response is contained in $\Phi_{0}$, every selection must be decreasing. Now, in any symmetric equilibrium with $n \geq n_{0}$ it holds that

$x=\frac{y}{n-1} \leq \frac{y+R}{n_{0}-1}$. That is, any symmetric equilibrium with $n \geq n_{0}$ firms occurs in $\Phi_{0}$. In this region, the best response is strongly decreasing. Uniqueness of any symmetric equilibrium follows from identical arguments made in the proof of Proposition 1. The comparative statics in parts (i) - (iii) follow from similar arguments as made in the proof of Propositions 2, 3, and 4 .

\section{Proof of Proposition 9}

Fix $z \geq 0$. It suffices to show that $\lim _{n \rightarrow \infty} P^{\prime}(z)+\frac{z}{n} P^{\prime \prime}(z)<0$. But $\lim _{n \rightarrow \infty} P^{\prime}(z)+\frac{z}{n} P^{\prime \prime}(z) \leq \lim _{n \rightarrow \infty}-\epsilon+\frac{z}{n} B=-\epsilon<0$.

\section{References}

Acemoglu, D. and Jensen, M. K. (2013). Aggregate comparative statics. Games and Economic Behavior, 81:27-49.

Amegashie, J. A. (1999). The number of rent-seekers and aggregate rentseeking expenditures: an unpleasant result. Public Choice, 99(1):57-62.

Amir, R. (1996). Cournot Oligopoly and the Theory of Supermodular Games. Games and Economic Behavior, 15(2):132-148.

Amir, R. and Lambson, V. (2000). On the effects of entry in Cournot markets. Review of Economic Studies, 67(2):235-254. 
Bamón, R. and Frayssé, J. (1985). Existence of Cournot equilibrium in large markets. Econometrica, 53(3):587-598.

Baye, M. R., Kovenock, D., and De Vries, C. G. (1993). Rigging the lobbying process: An application of the all-pay auction. The American Economic Review, 83(1):289-294.

Chowdhury, S. M. and Sheremeta, R. M. (2011). Multiple equilibria in Tullock contests. Economics Letters, 112(2):216-219.

Corchón, L. C. (2007). The theory of contests: A survey. Review of Economic Design, 11(2):69-100.

Cornes, R. and Hartley, R. (2005). Asymmetric contests with general technologies. Economic Theory, 26(4):923-946.

Dixit, A. (1987). Strategic behavior in contests. The American Economic Review, pages 891-898.

Fang, H. (2002). Lottery versus all-pay auction models of lobbying. Public Choice, 112(3-4):351-371.

Gama, A. and Rietzke, D. (2017). Robust comparative statics in contests. Lancaster University, Department of Economics Working Paper Series, No. 2017/017.

Jensen, M. K. (2010). Aggregative games and best-reply potentials. Economic Theory, 43(1):45-66.

Jensen, M. K. (2016). Existence, uniqueness, and comparative statics in contests. In Equilibrium Theory for Cournot Oligopolies and Related Games, pages 233-244. Springer.

Konrad, K. (2009). Strategy and Dynamics in Contests. Oxford University Press, New York, NY.

Loury, G. C. (1979). Market structure and innovation. The Quarterly Journal of Economics, pages 395-410. 
Matros, A. (2006). Rent-seeking with asymmetric valuations: Addition or deletion of a player. Public Choice, 129(3-4):369-380.

Menicucci, D. (2006). Banning bidders from all-pay auctions. Economic Theory, 29(1):89-94.

Milgrom, P. and Roberts, J. (1990). Rationalizability, learning, and equilibrium in games with strategic complementarities. Econometrica, 58:12551277.

Novshek, W. (1980). Cournot equilibrium with free entry. The Review of Economic Studies, 47(3):473-486.

Novshek, W. (1985). On the existence of Cournot equilibrium. The Review of Economic Studies, 52(1):85-98.

Nti, K. O. (1997). Comparative statics of contests and rent-seeking games. International Economic Review, 38(1):43-59.

Perez-Castrillo, J. D. and Verdier, T. (1992). A general analysis of rent-seeking games. Public Choice, 73(3):335-350.

Seade, J. (1980). On the effects of entry. Econometrica, 48(2):479-489.

Skaperdas, S. (1996). Contest success functions. Economic Theory, 7(2):283290.

Szidarovszky, F. and Okuguchi, K. (1997). On the existence and uniqueness of pure Nash equilibrium in rent-seeking games. Games and Economic Behavior, 18(1):135-140.

Topkis, D. (1978). Minimizing a submodular function on a lattice. Operations Research, 26(2):305-321.

Tullock, G. (1980). Efficient rent-seeking. In Buchanan, J. M., Tollison, R. D., and Tullock, G., editors, Toward a theory of the rent-seeking society, number 4, pages 97-112. Texas A\&M University Press, College Station, Texas. 
Vives, X. (1990). Nash equilibrium with strategic complementarities. Journal of Mathematical Economics, 19(3):305-321.

Vives, X. (1999). Oligopoly Pricing: Old Ideas and New Tools. MIT Press, Cambridge, MA.

Vives, X. (2005). Complementarities and games: New developments. Journal of Economic Literature, 43(2):437-479.

Vives, X. (2008). Innovation and competitive pressure. The Journal of Industrial Economics, 56(3):419-469.

Yamakazi, T. (2008). On the existence and uniqueness of pure-strategy nash equilibrium in asymmetric rent-seeking contests. Journal of Public Economic Theory, 10(2):317-327. 